\title{
Improving the search for victims in mountain environments with geovisualization and competing hypotheses management
}

\author{
Matthieu Viry ${ }^{\mathrm{a} *}$, Marlène Villanova-Oliver ${ }^{\mathrm{a}}$, Jacques Gautier ${ }^{\mathrm{a}}$, Matthew Sreeves ${ }^{\mathrm{a}}$, Paule-Annick \\ Davoine ${ }^{\mathrm{a}, \mathrm{b}}$
}

${ }^{a}$ Univ. Grenoble Alpes, CNRS, Grenoble INP, LIG, 38000 Grenoble, France, matthieu.viry@univ-grenoble-alpes.fr, marlene.villanova-oliver@univ-grenoble-alpes.fr. matthew.sreeves@univ-grenoble-alpes.fr.jacques.gautier@univ-grenoble-alpes.fr ${ }^{b}$ Univ. Grenoble Alpes, CNRS, Sciences Po Grenoble, Pacte, 38000 Grenoble, France, paule-annick.davoine@univ-grenoble-
alpes.fr

* Corresponding author

\begin{abstract}
:
This article is part of a research program aimed at improving the location of victims in the mountains. The search for victim location is a process involving various elements, few of them falls within the scope of our team such as the modelling of the process of spatial reasoning of the rescuer and the geovisualization of multidimensional and uncertain information for decision-making. After having reviewed the various scientific challenges we propose a prototype of interface. The strengths of our approach are the transformation of location clues into a list of spatial filters, the addition of these filters within a search scenario to create probable location area(s) as well as the simultaneous management of competing search scenarios. We also present the results of user tests, validating the interest of such an approach to objectify the area of probable location of the victim and obtain a more precise area than during the traditional search approach. Finally, we will discuss the improvements underway on the basis of this prototype, in particular how we plan to better account for the uncertainty in the decision-making process and how we plan to allow a future prototype to suggest questions to the rescuer in order to help him/her refine his/her research.
\end{abstract}

Keywords: geovisualization, spatial reasoning, decision support, search and rescue.

\section{Introduction}

In certain cases where no technology such as GNSS is available, mountain rescue workers who receive an emergency call have to process by their own to localise the victim. The research phase starts with the exploitation of what the caller (the person who triggers the alert, who is not necessarily the victim) says, trying to describe where $\mathrm{s} /$ he is currently and/or which path $\mathrm{s} / \mathrm{he}$ has followed till there. An example of such descriptions is "I left two hours ago from the Refuge de la $\mathrm{Pra}^{l}$ and walk in the direction of the Cascade de l'Oursière. Now, I see partly a lake just below". All these pieces of information are clues analyzed by the rescuer who looks in different data sources for corresponding elements that could help to localize the victim. Data sources may be printed or digital maps or guidebooks. The alert processing is also

1 in French in the text particularly leverage on the expertise of the rescue workers and their knowledge of the terrain. Several elements complicate the localization task: the caller often describes the current position and the path followed with imprecise relative positions in relation to sometimes ambiguous reference objects (ambiguity can be in the naming of these objects, in their description, in the expression of the relative position itself). The data needed by rescuers for localization are heterogeneous, multisource, dispersed and insufficiently structured so they are efficiently queried. If the search problem is necessarily spatial as "many decisions taken by both the subject and rescuers are influenced by the terrain" (Michal \& Robert, 2015), it may obviously benefit from a better exploitation of the information collected during the alert call. This is the starting point of the project CHOUCAS funded by the French National Research Agency. The whole project addresses multiple scientific 
challenges (Olteanu-Raimond et al, 2017): data structuring from heterogeneous textual sources, integration of spatialisable heterogeneous sources, fuzzy qualitative spatial reasoning, geovisualization of multidimensional and uncertain data for decision making, etc. This paper focuses on our work related to the development of a geovisualization-based approach for supporting rescuer's spatial reasoning process when handling an emergency call. Our objectives are to help rescue workers i) to capture clues get from the call so to ease their processing, ii) to take them into account while building/exploring/refining hypotheses about the victim's localization, and ii) to visualize the probable location areas compatible with their hypotheses.

This paper is organized as follows. In section 2 we present the requirements for a geovisualization solution faces the challenges presented above before we present some existing works in section 3. An overview of our prototype is given in section 4 . We describe in section 5 first experiments we have conducted with users. Finally, in section 6, we will discuss the lessons to be learned from these experiments in terms of improvements that could be implemented in a future prototype.

\section{Requirements for a geovisualization approach that supports rescuers' reasoning process}

\subsection{Introducing example}

Basically, the reasoning process of finding the victim consists in formulating hypotheses from the first information, sometimes incomplete or inaccurate, provided by the caller, and gradually refining them by taking into account additional clues to deduce and infer the probable location of the victim.

The following example introduces spatial (but not only) clues obtained successively by the rescue worker. It corresponds to a real situation that serves as common case study. For simplicity, we only give here expressions that depict how the rescuer interpreted information said by the caller as clues (please refer to (Bunel et al., 2019) to learn more about how we identify reference features and spatial relations used in mountain emergency calls). All clues are collected in the order given below. The victim:

- left Le Bourg d'Oisans on a pedestrian path.

- $\quad$ walked several hours towards a ski station.

- felt several meters.

- sees part of a body of water.

- is below a road and hears vehicles.

- is beneath an electric line.

- $\quad$ was in the sun but is now in the shade.
In this study case, except the name of the starting town (Le Bourg d'Oisans), no information is given that could help to identify known reference objects on the field: no pedestrian path number, no name for the ski station or for the body of water that is here moreover poorly described. Few decision-support tools are available to assist rescue workers in processing such clues in such a context for determining a likely location of the victim (see section 3). Our hypothesis is that an interactive geovisualization tool could facilitate the localization of victims by supporting rescuers spatial reasoning while accounting for the uncertainty of the information being mobilized and allowing them to explore several competing hypotheses.

\subsection{Hypothesis formulation}

The reasoning process of the rescue worker can be assimilated to a hypotetico-deductive model applied several times: at each step of the reasoning, s/he formulates a hypothesis about the localization matching spatially with the clues collected. For instance, in our case study the first hypothesis made is that the victim has to be searched in an (large) area drawn around the center of the town Le Bourg d'Oisans, from where pedestrian paths start. At this stage, no other assumption can be made as nothing is said about the fact that the victim is still on such a hiking path or not. Then, in the reasoning process, this first area is confronted to the new clues collected, expecting these will contribute step by step to reduce spatially the possibilities. The information "walked several hours towards a ski station" here helps to refine the location hypothesis considering where are the ski stations with regard to Le Bourg d'Oisans. So, our underlying assumptions are:

- the rescuer tries to translate each information given (when possible) into a spatial relation implying objects of reference that then acts as a spatial clue in her/his reasoning process;

- this reasoning process relies on successive hypotheses that correspond to probable localization areas for the victim, built considering spatial clues.

- each successive localization hypothesis is constructed as the spatial intersection of the areas corresponding to spatial clues.

The reasoning process of the rescuers is made complex in several ways. First, the collect itself of information can be difficult due to the physical and psychological state of the victim; second, the transformation of information in spatial clues is not always a direct translation but sometimes requires to manage indirect referencing (e.g. "the victims is below a road"), or inter-visibility situations; third the spatial clues can lead to several distinct areas to be considered which can result in several 
competing localization hypotheses to explore; four, the imperfect nature of the information provided by the caller (see section 2.3) and/or its credibility as perceived by the rescuers has to be consider. This requires means to explore competing localization hypotheses offering this way the possibility of pursuing different scenarios. Geovisualisation tool can be of great interest here in line with (Andrienko et al., 2007) for whom an efficient geovisual analytics and spatial decision tool should supports to "create plausible scenarios representing possible courses of action, compute and visualize their impacts, test the stability of scenarios, and prioritize them to help select the right course of action".

\subsection{Imperfect information}

As stated before, information get by the rescuer as well as the probable areas of location that may emerge from her/his reasoning may be imperfect in nature. Previous studies have defined taxonomies of imperfect information. The framework given by (Snoussi \& Davoine, 2014) and adapted by (Saint-Marc, 2017) distinguishes three types of imperfect information, which can also be applied to the search for victims:

- imprecision, where "the true value is located in a defined subset of values", corresponds to situations where the victim is next to a body of water but cannot tell which one among a set of bodies of water present in the area.

- inconsistency, which is defined as a "conflict or incoherence in the values", matches situations of incoherence in victims' descriptions,.

- uncertainty, which is defined as a "partial knowledge about the true value of information", corresponds to the difficulty of precisely qualifying the distance separating the victim from an object for example.

\section{Existing work}

Geovisual Analytics is interested in the cognitive processes implemented during the visual analysis of dynamic and/or interactive cartographic support. It frequently refers to the analysis of large volumes of data, for example to see the emergence of major trends (Robinson et al., 2017). In our case, it is rather a matter of guiding the rescuer towards effective decision-making and getting him/her to explore other research options.

According to Andrienko et al., Geovisual Analytics for Spatial Decision Support "covers all phases of the decision-making process: analyzing the situation of the problem, finding or constructing possible solution options and evaluating these options in order to select the best of them" (Andrienko et al., 2007), and they highlight several points that match to our concerns, including:
- enabling rational decision-making,

- enabling decision-making in an emergency context,

- allowing heterogeneous information to be taken into account.

The representation of imperfect information and its effect on decision-making has been cited for several years as a challenge in terms of information visualization and geovisualization (Çöltekin et al., 2017). Proposals regarding its representation are numerous (MacEachren, 1992; MacEachren et al., 2005; Deitrick \& Wentz, 2015; Koo et al., 2018) but not all of them seems to be adopted as unanimously as other traditional semiology features.

Many of these proposals concern only a punctual representation of uncertainty (Berjawi et al., 2014), which is not suitable for surface representations. When they concern surfaces, these works generally address the level of confidence of a predicted phenomenon (MacEachren et al., 2005) or a variable calculated for a given administrative territory (Koo et al., 2018) while we are particularly interested in the calculation and the uncertain representation of an area for which neither the limits nor the location are known a priori.

Representations of uncertainty and their impacts on decision-making have been studied (Kinkeldey et al., 2017), in order to be able to characterize various effects such as decision performance, decision outcome and decision confidence. Recent works in the field of aeronautical search and rescue operations (Riveiro et al., 2016) study decision-making with a geovisual and uncertain representation of positioning data coming from sensors. In particular, this study tells us that the representation of uncertainty did not increase the workload of the participants nor the time required for classification tasks. Moreover, uncertainty representation seems to have a variable effect on the confidence of the classification decisions made by the operators according to their expertise's level: half of the experts are more confident when the uncertainty is displayed whereas no novice made this comment.

Although experts seem less influenced by the representation of uncertainty than novices (Aerts et al., 2003; Riveiro et al., 2016) the effects of the level of expertise on decision-making in a context of uncertainty are still poorly understood. Notably, we claim that it is crucial to choose representations that lead neither to underestimate nor overestimate data uncertainty, which in both cases would be counterproductive.

Several examples of application prototypes that try to tackle one or more of theses challenges have been developed and tested in recent years. While they share the use of geovisualization for decision support, they deal 
with a variety of themes: epidemiology (Robinson et al., 2005), resilience of maritime surveillance system (Vatin, 2014), aeronautical search and rescue operations (AbiZeid et al, 2010), understanding of spatial dynamics of flood events management (Saint-Marc, 2017).

Closer to our objectives, a specific approach to Search and Rescue (S\&R) of lost people is described in (Michal \& Robert, 2015). This approach, developed as an extension to a desktop GIS application, focuses on the modeling of lost persons behaviors from their last known position and their point of destination. More recently, in October 2018, a complete solution dedicated to S\&R operations of missing people have emerged (SHARON tool $^{2}$ ) allowing to estimate the search area of a victim in an integrated environment. An originality of this solution is to make suggestions for the allocation of research units based on their real-time locations.

Our approach is original and innovative: it proposes to combine the visualization of geospatial elements for decision support, the creation of one or more search scenarios while trying to take into account the imperfect nature of the information.

\section{The prototype for supporting the spatial reasoning process}

\subsection{Overview}

In order to meet the needs mentioned in section 2, we propose a prototype of a victim location system (Fig. 1) whose originality and innovation are to combine the visualization of geospatial elements for decision support, the creation of one or more search scenarios while taking into account the imperfect nature of the information.

This prototype proposes to create and combine simple search areas, corresponding to clues get from the caller, in an interface that allows the rescuer to manage and manipulate these clues in order to build one or more localization hypotheses (called scenarios in the interface). The different elements of this interface are the following: - The main zone composed of a base map (on the right of Fig. 1) that gives a spatial representation of the situation (i.e. hypotheses built).

- A list of objects, classified in a tree, allowing the selection of a given object or of a category of objects (for example: "all lakes") to what refers the information given by the caller.

- An interactive table that captures the clues elaborated by the rescuer from what $\mathrm{s} /$ he gets during the call and offers the means to build and compare competing localization hypotheses.

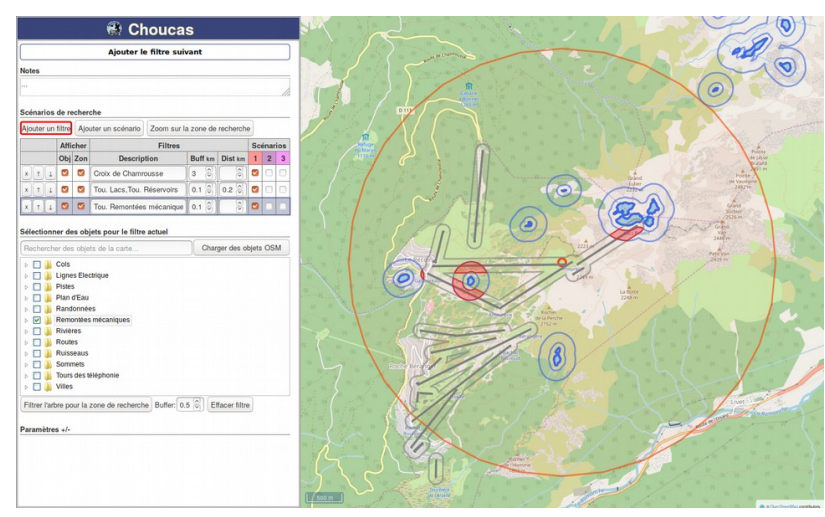

Fig 1. Overview of the prototype interface.

These three components are interconnected between them: the objects chosen in the tree can be added as input in the table of clues; clues in the table that participate in a scenario are represented on the map, which more over renders the potential localization of the victim as the intersection of spatial extents of these clues. For instance, in Fig. 1, the map depicts the situation that corresponds to a scenario in which three clues given as input i.e. to be: 1) at around $3 \mathrm{~km}$ for the submit known as "Croix de Chamrousse"; 2) at the immediate vicinity of a body of water; 3) under a ski lift. These clues lead to the identification of four probable areas (polygons in red).

In the next sections, we detail the possibilities offered by our prototype for supporting the reasoning process of the rescuer: the Table of filters allows for managing clues and managing hypothesis; the searchable item tree to select the reference spatial objects.

\subsection{Table of filters}

The filter management panel (Fig. 2) is the central element in the management of search clues by the rescuer.

\begin{tabular}{|c|c|c|c|c|c|c|c|c|c|c|}
\hline & & & \multicolumn{2}{|c|}{ Afficher } & \multicolumn{3}{|l|}{ Filtres } & \multicolumn{3}{|c|}{ Scénarios } \\
\hline & & & Obj & Zon & Description & Buff km & Dist km & 1 & 2 & 3 \\
\hline$x$ & $\uparrow$ & $\downarrow$ & $\nabla$ & $\theta$ & Croix de Chamrousse & $3 \hat{v}$ & $\hat{i}$ & $\theta$ & $\square$ & 0 \\
\hline $\mathrm{x}$ & $\uparrow$ & $\downarrow$ & $\theta$ & $\theta$ & Tou. Lacs, Tou. Réservoirs & $0.1 \hat{\sim}$ & $0.2 \hat{\sim}$ & $\varnothing$ & $\square$ & $\square$ \\
\hline $\mathrm{x}$ & $\uparrow$ & $\downarrow$ & $\nabla$ & $\nabla$ & Tou. Remontées mécanique & $0.1 \hat{\sim}$ & $\hat{v}$ & $\nabla$ & $\square$ & $\square$ \\
\hline
\end{tabular}

Fig. 2. An example of table of filters filled. The table currently contains 3 filters to express clues as mentioned in section 4.1.

Each row of the table corresponds to a clue built from the caller's information. The columns to the right of each line correspond to the search hypotheses (scenarios) in which these clues are mobilized or not. In Fig. 2, only one scenario appears, composed of the combination of three clues retained as filters. Whenever the rescuer doubts from the veracity of a clue, s/he can easily build a new scenario in which s/he unchecks the corresponding box (and keeps the others checked). By clicking on the number of the scenario, the user sees on the map the

2 http://www.eledia.org/showcase/sharon/ 
representation for the probable localization resulting from this scenario.

This illustrates the flexibility offered by our prototype to build and compare hypotheses. Indeed, the user can add / remove filters, reorder them and decide to include them, temporarily or throughout the analysis, in one or more research hypotheses. S/he can also, for each of these filters, choose to display the object(s) and/or the corresponding created zone(s) or not on the map. In the next sections, we present how the clues are acquired using the searchable items tree and how to express some uncertainty.

\subsection{Searchable items tree}

The searchable items tree (Fig. 3) proposes a list of objects extracted from OpenStreetMap and organized into thematic groups: mountain pass, power lines (3 cables, 6 cables, others), ski runs (blue, black, green, red), bodies of water (lakes, small bodies of water, reservoirs), hiking trails, ski lifts, rivers, roads, streams, peaks, telephony towers and cities.

\begin{tabular}{l} 
Sélectionner des objets pour le filtre actuel \\
\hline Rechercher des objets de la carte... \\
$\qquad \square$ Cols \\
$\triangleright \square$ Lignes Electrique \\
$\triangleright \square$ Pistes \\
$\triangleright \square$ Plan d'Eau \\
$\triangleright \square$ Randonnées \\
$\triangleright \square$ Remontées mécaniques \\
$\triangleright \square$ Rivières \\
$\triangleright \square$ Routes \\
$\triangleright \square$ Ruisseaus \\
$\triangleright \square$ Sommets \\
$\triangleright \square$ Tours des téléphonie \\
$\triangleright \square$ Villes \\
Filtrer l'arbre pour la zone de recherche Buffer: $0.5 \hat{\sim}$ Effacer filtre
\end{tabular}

Fig. 3. Searchable items tree.

It allows the user to select objects mentioned by the caller, to create clues that serve as filters as described above. Objects can be added individually (e.g. in Fig. 2 "Croix de Chamrousse" which is a sub-item of the entry "Sommets" - peaks) or by group (e.g. in Fig. 2, "Tou. Remontées Mécaniques" - all ski lifts).

This list of objects is backed by a text search bar allowing the user to start typing the name of an object s/he is looking for: the list is automatically filtered according to the characters that are being entered. The chosen object(s) are then added to the table of filters.

\subsection{Taking into account distance and approximating uncertainty}

When creating a filter, after selecting an object or an object group, the user can enter one or two distance information (Fig. 2, respectively columns Buff and Dist):
- "buffer" (in kilometers) to choose the size of the zone to create around an object to render the possible location of the victim relatively to that object;

- "distance" (in kilometers) to model the case where the victim is at some distance from an object: this field creates a line around an object or type of object at the desired distance, the value of "buffer" chosen is then applied to this line rather than to the object itself.

These two input fields can be used to express information such as the victim is about between 500 to 800 meters from a lake: the buffer is put to $0.3 \mathrm{~km}$ and the distance to $0.5 \mathrm{~km}$. This offers some means to transcript uncertainty about distances.

Each time a filter is specified, the map is updated to represent the corresponding clue and its impact on the probable location(s) of the victim.

\subsection{Representing clues and probable location areas}

Fig. 4 shows the map resulting from the scenario previously described.

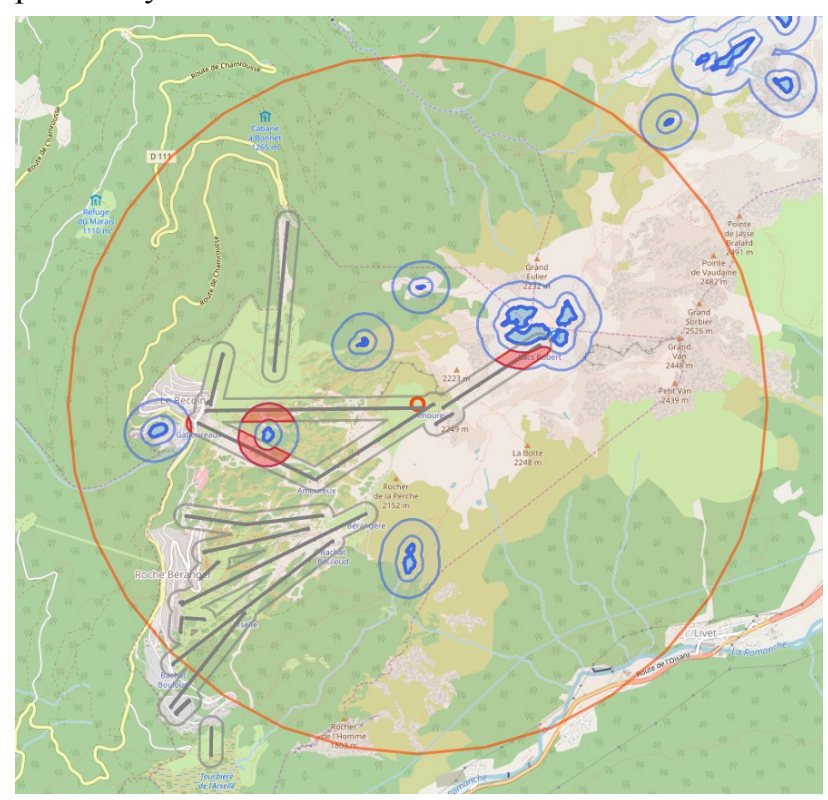

Fig. 4. Displaying of probable location areas for the case depicted in section 4.1 (each kind of object is outlined in a specific color, the probable location areas are filled in the color of the scenario).

The objects and areas that correspond to each of the mobilized clues have their outlines in a color associated with the type of object concerned: the small circle in orange at the center of the map represents the Croix de Chamrousse with a buffer around (big circle also in orange); bodies of water and their buffer/distance elements are represented in blue; ski lifts (objects and buffers) are in black. One can notice that overlapping polygons associated with the same type of objects are merged.

Probable areas of location are the ones calculated as the intersection of polygons of the three kinds (i.e. the ones 
corresponding to the three clues). Four areas are found. They are filled with the color corresponding to the scenario in the interface (here in red). Left to right on Fig 4, we found one very small at an extremity of the ski lift, then two around the same lake, and the last on the other side of the Croix de Chamrousse. Such visualization is useful for the user to orientate the discussion with the caller in order to find new clue. This point is discussed in section 6 .

\section{Experimentation}

The prototype has been tested on two high-mountain rescuers and on five other users (non-rescuers but experts of geographic information and comfortable with the manipulation of this kind of interface).

Users were first proposed to perform a tutorial (a simplified case of research, in which the user is guided on the various panel to use) followed by the processing of the red thread scenario (see section 2.1). In both cases, the goal is to translate the successive clues in the interface and to define the probable location of the victim. We observed the users during both parts of this test and we collected their feedback.

The testers highlighted positive points of our tool, notably regarding to the items tree. First, the practical side of the items tree displayed is underlined as well as the fact that it can be filtered to match the viewport. Second, the overview of all the instances available for a type of object is appreciated (as it for instance allows not to miss a body of water that a rescuer might not know). Third, they endorse that, unlike the system used so far, our prototype makes it possible to display areas of probable location, based on the intersection of the zones relative to each clue, and to manually draw specific zones to indicate the possible presence of the victim.

In comparison with the system used previously, these different points make it easier to identify a probable location area and then to precisely define a zone, which is especially true for people who have no knowledge of the area (indeed rescuers have a strong business knowledge but don't always know the specific area of intervention).

Our test users also raised several negatives points :

- the lack of interactivity of the map (indeed the manipulation of the clues is only done through the items tree), which caused a loss of the clues spatial context with one of the rescuer, who abandoned his knowledge of the field to focus only on the items tree.

- the difficulty to model some situations with the proposed combo "buffer" + "distance" + "object" (or "group of objects") to translate what is said by the victim.

- some performance problems (expensive operations, such as intersections, on a sometimes high number of
OpenStreetMap objects) added to the absence of some indicator that the user interface is currently busy.

- the hierarchical structure of the items tree, which was seen as a positive point by the first testers, requires a certain level of expertise of the domain and abilities in vagueness or imprecision exploitation to manage situation in which objects have to be found in the tree (for instance, victims will not refer to a "water body" by "reservoir" and vice versa).

The two rescuers did not have the same strategy when they are facing the prototype's interface. One was focused on the translation of every clue into buffered zones in order to generate intersected areas, and the other used more often his knowledge of the area, in order to visually select, on the map, which items were trustworthy. Therefore we should not expect only one type of behavior but explore various behaviors or various research strategies.

Furthermore it is interesting to note that the clue "from Bourg-d'Oisans and to a ski resort" doesn't explicitly specify which is the ski resort. One of the rescuers, despite the fact he was mentally thinking and exploring the possible implications of two probable ski stations (which are located at two opposite sides from the victim's starting point), did not consider himself to be "exploring several scenarios".

These first observations confirm the interest of helping the rescuer's spatial reasoning with the management of clues, while the management of hypotheses seems of less priority. It also confirms that we must go further in the consideration of uncertainty (which is here approached only by the value "buffer" in the interface). We are also encouraged to keep on investigating how to support the reasoning of the rescuer, without being invasive in the interface.

\section{Discussion}

\subsection{Imperfect information management}

The calculation method for determining probable location areas will be refined within the project to consider more options than the simple intersection we use and to include more complex expression of imperfection. Currently the imperfection of the information provided is approximated through the buffer parameter used when creating the filters. We are working on new input components to allow the rescuer to handle more efficiently this uncertainty within the interface.

Another form of uncertainty that is not currently being used is the level of confidence that the rescuer lends to the information provided by the caller. We think it is necessary that, in addition to taking into account the uncertainty, the rescuer can note the level of confidence 
$\mathrm{s} /$ he has regarding information. This level of confidence could be used later in various ways that remain to be determined: as a weighting during the calculation of probable location area, to seek for elements that would help to reduce the uncertainty regarding to the location of the victim (see next paragraph), to highlight a strong or a weak trust on some clue on the map, etc.

\subsection{Guided information collect for the rescuer}

One possible improvement for the rescuer would be to suggest him/her what to ask to the caller considering elements present in the search area(s) in order to obtain new clues and refine the probable location of the victim.

Indeed, in the case shown in Fig. 4, some of the probable localization zones contain distinctive and highly discriminating elements (distinct land-uses, presence of roads in one of the zones, presence of a refuge near one of the other zones). This information could be relayed by our system to the rescuer to try to obtain information about these elements: "Are you near a road ?", "Do you ear vehicles ?", "Have you been near a shelter ?".

Moreover, to model more easily the reasoning of the rescuer when obtaining such information, our system should provide an inverse functionality to the current creation of filters in order to allow the rescuer to manage the fact that a specific object is not seen by the victim or is not near him/her.

\subsection{Automatic loading of objects in the current area}

Currently the loading of the objects corresponding to a zone, in order to fill the searchable items tree, is done by a click on the button "loading of the objects of the zone" (which entails a request to the Overpass $\mathrm{API}^{3}$ ) while the background map is proposed in the form of an assembly of raster tiles (images sent on demand to the client in PNG format). One possible improvement could be the use of vector tiles to replace current raster tiles. This would make it possible to have a direct knowledge of the OpenStreetMap objects present on the displayed zone or in the probable location zone, thus saving the delay of the request releasing the rescuer of the responsibility of clicking on a button to carry out this request. This improvement, based on recent technologies, would free the rescuer of a logic of data recovery to benefit from a logic of data exploitation (Noucher, 2009).

Unlike raster tiles, vector tiles are not static images, but extracts from a dataset: they contain the geometries, attribute data and metadata of the different objects. The style is applied to it on the fly, in the client's web browser, when receiving each tile. So the style is independent of the data itself. Vector tiles are typically smaller than raster tiles for better resolution, better loading speeds, and efficient caching (Hayat, 2017).

\subsection{Visual and interactive management of competing scenarios in addition to better map interaction}

A new version of the prototype we are working on aims to graphically represent the clues and the fact that they are mobilized in one or more scenarios. This representation of the clues is expected to be closer to how the rescuer organizes them intuitively and operates them in scenarios. An effort will be put on possibilities to interactively modify clues to create several search scenarios with slight differences (for example a scenario with only the specific lake cited by the applicant and a second scenario with the all the lakes in the area). This will enable these different scenarios to be effectively confronted using ad hoc interactive and synchronized map representations. This could bring out more efficiently areas of probable locations or areas in which to seek for additional discriminating elements on which to interrogate the caller.

\section{Acknowledgements}

This work is supported and financed by French National Research Agency (ANR) under the CHOUCAS project (ANR-16-CE23-0018).

\section{References}

Abi-Zeid, I., Nilo, O., Schvartz, S., \& Morin, M. (2010). Towards a knowledge-based system prototype for aeronautical Search and Rescue operations. In 2010 13th International Conference on Information Fusion (pp. 1-8). Edinburgh: IEEE. https://doi.org/10.1109/ICIF.2010.5712112

Aerts, J. C. J. H., Clarke, K. C., \& Keuper, A. D. (2003). Testing Popular Visualization Techniques for Representing Model Uncertainty. Cartography and Geographic Information Science, 30(3), 249-261. https://doi.org/10.1559/152304003100011180

Andrienko, G., Andrienko, N., Jankowski, P., Keim, D., Kraak, M.-J., MacEachren, A., \& Wrobel, S. (2007). Geovisual analytics for spatial decision support: Setting the research agenda. International Journal of Geographical Information Science, 21(8), 839-857. https://doi.org/10.1080/13658810701349011

Berjawi, B., Duchateau, F., Chesneau, É., Favetta, F., Seccia, G., Cunty, C., ... Laurini, R. (2014). Uncertainty visualization of multi-providers cartographic integration. Journal of Visual Languages \& Computing, 25(6), 995-1002. https://doi.org/10.1016/j.jvlc.2014.10.033

3 The Overpass API is a read-only API that serves up custom selected parts of the OpenStreetMap data upon client queries. 
Bunel, M., Duchêne, C., Olteanu-Raimond, A-M., Villanova-Oliver, M., Bonhoure, G., Jouan, T., An approach for identifying and analysing reference features and spatial relations used in mountain emergency calls. ICC'2019, July 15-20, 2019, Tokyo, Japan.

Çöltekin, A., Bleisch, S., Andrienko, G., \& Dykes, J. (2017). Persistent challenges in geovisualization - a community perspective. International Journal of Cartography, 3(sup1), 115-139. https://doi.org/10.1080/23729333.2017.1302910

Deitrick, S., \& Wentz, E. A. (2015). Developing Implicit Uncertainty Visualization Methods Motivated by Theories in Decision Science. Annals of the Association of American Geographers, 105(3), 531-551. https://doi.org/10.1080/00045608.2015.1012635

Hayat, F. (2017). Translation from the collaborative OSM database to cartography. In Proceedings of the 2017 International Cartographic Conference. Washington, D.C., United States. https://doi.org/10.5194/ica-proc-1$\underline{53-2018}$

Kinkeldey, C., MacEachren, Alan M., Riveiro, M., \& Schiewe, J., (2017). Evaluating the effect of visually represented geodata uncertainty on decision-making: systematic review, lessons learned, and recommendations. Cartography and Geographic Information Science, 44(1), 1-21. https://doi.org/10.1080/15230406.2015.1089792.

Koo, H., Chun, Y., \& Griffith, D. A. (2018). Geovisualizing attribute uncertainty of interval and ratio variables: A framework and an implementation for vector data. Journal of Visual Languages \& Computing, 44, 89-96. https://doi.org/10.1016/j.jvlc.2017.11.007

MacEachren, Alan M. (1992). Visualizing Uncertain Information. Cartographic Perspective, (13), 10-19. http://dx.doi.org/10.14714/CP13.1000

MacEachren, Alan M., Robinson, A., Hopper, S., Gardner, S., Murray, R., Gahegan, M., \& Hetzler, E. (2005). Visualizing Geospatial Information Uncertainty: What We Know and What We Need to Know. Cartography and Geographic Information Science, 32(3), 139-160. https://doi.org/10.1559/1523040054738936

Michal, W., \& Robert, M. (2015). DECISION SUPPORT SYSTEM FOR SEARCH \& RESCUE OPERATIONS. Computer Science, 16(3), 281. https://doi.org/10.7494/csci.2015.16.3.281

Noucher, M. (2009). La donnée géographique aux frontières des organisations: approche socio-cognitive et systémique de son appropriation. (Theses). Ecole
Polytechnique Fédérale de Lausanne (EPFL). Retrieved from https://tel.archives-ouvertes.fr/tel-00654203

Olteanu-Raimond, A.-M., Davoine, P.-A., Gaio, M., gouarderes, eric, Van Damme, M.-D., VillanovaOliver, M., ... Ziebelin, D. (2017). Projet CHOUCAS: Intégration de données hétérogènes et raisonnement spatial pour l'aide à la localisation des victimes en montagne. In Spatial Analysis and GEOmatics 2017. Rouen, France: INSA de rouen. Retrieved from https://hal.archives-ouvertes.fr/hal-01649156

Riveiro, M. (2016). Visually supported reasoning under uncertain conditions: Effects of domain expertise on air traffic risk assessment. Spatial Cognition \& Computation, 16(2), 133-153. https://doi.org/10.1080/13875868.2015.1137576

Robinson, A. C., Chen, J., Lengerich, E. J., Meyer, H. G., \& MacEachren, A. M. (2005). Combining Usability Techniques to Design Geovisualization Tools for Epidemiology. Cartography and Geographic Information Science, 32(4), 243-255. https://doi.org/10.1559/152304005775194700

Robinson, A. C., Demšar, U., Moore, A. B., Buckley, A., Jiang, B., Field, K., ... Sluter, C. R. (2017). Geospatial big data and cartography: research challenges and opportunities for making maps that matter. International Journal of Cartography, 3(sup1), 32-60. https://doi.org/10.1080/23729333.2016.1278151

Saint-Marc, C. (2017). Formalization and geovisualization of historical natural risk events to understand spatial dynamics (Theses). Université Grenoble-Alpes. Retrieved from https://hal.archivesouvertes.fr/tel-01572326

Snoussi, M., \& Davoine, P.-A. (2014). Methodological proposals to handle imperfect spatial and temporal information in the context of natural hazard studies. Revue Internationale de Géomatique, 3-4(23), 495-517. https://doi.org/10.3166/rig.23.495-517

Vatin, G. (2014). Formalization of a Geovisual Analytics Support Environment (Theses). Ecole Nationale Supérieure des Mines de Paris; CRC - Centre de recherche sur les Risques et les Crises. Retrieved from https://tel.archives-ouvertes.fr/tel-01253688 